\title{
Quaderni
}

QUADERNI Communication, technologies, pouvoir

86 | Hiver 2014-2015

Penser la politique par le film

\section{Marie Veniard, La nomination des événements dans la presse. Essai de sémantique discursive}

\section{Christine Barats}

\section{OpenEdition}

1 Journals

\section{Édition électronique}

URL : http://journals.openedition.org/quaderni/871

DOI : 10.4000/quaderni.871

ISSN : 2105-2956

Éditeur

Les éditions de la Maison des sciences de l'Homme

\section{Édition imprimée}

Date de publication : 5 janvier 2015

Pagination : 83-86

\section{Référence électronique}

Christine Barats, « Marie Veniard, La nomination des événements dans la presse. Essai de sémantique discursive », Quaderni [En ligne], 86 | Hiver 2014-2015, mis en ligne le 05 janvier 2017, consulté le 25 septembre 2020. URL : http://journals.openedition.org/quaderni/871 ; DOI : https://doi.org/10.4000/ quaderni.871 


\section{Compte rendu}

\section{La nomination des événements dans la presse. Essai de sémantique discursive. Marie Veniard}

Besançon, Presses universitaires de Franche-Comté, 2013

par Christine Barats

Université Paris Descartes

Céditec, Université Paris Est Créteil

1. A savoir Le Monde et Le Figaro, choisi pour leur diffusion similaire.
« Guerre contre le terrorisme », « guerre en Afghanistan », " guerre contre Al Qaïda », « conflit », « crise »... autant de mots pour nommer l'événement et participer à la construction du sens social. Comment saisir les ressorts linguistiques, sémantiques et discursifs de cette configuration du sens ? C'est à une analyse minutieuse et rigoureuse de l'acte de nommer que nous convie Marie Veniard dans son ouvrage issu d'un travail de thèse mené en sciences du langage : La nomination des événements dans la presse. Essai de sémantique discursive. L'événement est appréhendé ici dans sa dimension langagière et discursive : l'événement ne fait pas que "perturber l'ordre normal des choses » lorsqu'il advient, il « engage les communautés concernées dans un travail sémantique » (p. 7) qui traduit les oppositions et contribue à les exercer. À partir d'un riche terrain d'observation, l'auteure examine la nomination d'un événement du point de vue de sa matérialité discursive. Ce n'est cependant pas uniquement une conception langagière de la nomination qui retient l'attention de l'auteure, mais également une conception anthropologique de l'acte de nommer. L'auteure s'inscrit ainsi dans une perspective pragmatique en examinant les luttes qui accompagnent l'acte de nommer. Elle propose d'ailleurs la notion de profil lexico-discursif pour dépasser la seule entrée lexicale et saisir le lexique en discours, faisant référence à la notion de dialogisme proposée par M. Bakhtine et à la façon dont on lutte avec et par le discours, renvoyant à la notion d'ordre du discours développée par Foucault (1971). L'auteure a retenu deux événements dans la presse quotidienne nationale ${ }^{1}$, deux conflits : un conflit armé, la guerre en Afghanistan (13 septembre 24 décembre 2001) et un conflit social, la grève des intermittents du spec- 
tacle (9 juin - 30 mai 2003). Elle a constitué deux corpus, respectivement de 3000 et 1500 articles (2 350000 occurrences et 1100000 occurrences) qui illustrent l'ouvrage en proposant de très nombreux exemples.

L'introduction et les deux premiers chapitres s'avèrent très utiles car ils explicitent la démarche adoptée. L'auteure souligne ainsi que « la configuration du sens social d'un événement (...) s'exerce à différents niveaux discursifs. Par le choix d'une catégorie plutôt que d'une autre, bien sûr ; par des effets sémantiques liés à la relation entre une dénomination et son cotexte; par la circulation des dires. » (p. 8). La nomination est ainsi au carrefour de ces trois niveaux discursifs : paradigmatique, syntagmatique et interdiscursif. Par exemple, le choix des termes « rebelles » ou « résistants » indique le point de vue adopté par rapport au conflit et rend compte des rapports de force qui s'exercent dans et par les discours. Le choix d'une préposition (« la guerre en Afghanistan » ou " contre l'Afghanistan ») éclaire également le travail de construction du sens, de même que la désignation ou la non désignation des protagonistes (p. 60). Citons également le choix de la rubrique dans laquelle s'insère l'article qui - comme par exemple dans le cas du conflit des intermittents du spectacle - peut varier et participer à inscrire l'événement dans la rubrique «Culture », « Politique » ou « Débats » (p. 61), ce qui contribue à la configuration du sens. Si la « désignation constitue le caractère principal du signe linguistique », la nomination est un " acte d'attribution qui relie une expression linguistique à un segment de réalité, qui livre un point de vue anthropologique sur l'objet nommé ». En insistant sur les différences terminologiques entre nomination, désignation et dénomination (p. 18), l'auteure rend accessible un vocabulaire qui peut parfois apparaître comme réservé aux seuls spécialistes des sciences du langage ou de la linguistique. Le chapitre 2 porte tout particulièrement sur les conflits discursifs et interprétatifs au moment de l'émergence de l'événement. L'étude du début du traitement médiatique des deux conflits met ainsi au jour l'importance de la temporalité dans le travail de configuration du sens. Dans le cas de la guerre en Afghanistan, l'auteure montre les difficultés à nommer l'événement : " guerre », " une seule bataille », « une longue campagne » (p. 38). Les logiques journalistiques de même que les enjeux désignatifs permettent d'observer les rapports de force en lice dans les difficultés de nommer l'adversaire. Ceci constitue un point commun aux deux conflits : dans le cas de l'Afghanistan, c'est la figure d'Oussama Ben Laden qui émerge et dans le cas des intermittents du spectacle c'est la quasi absence d'adversaire qui contribue à qualifier le conflit 
de suicidaire ou de grève contre soi-même (p. 42). Si les logiques de presse sont prises en compte dans l'analyse, l'examen de la circulation des différents syntagmes pour nommer la guerre et ses belligérants s'avère très intéressant pour comprendre les processus de circulation et d'anticipation observables dans les discours. Par exemple, dans le cas de la guerre en Afghanistan, la mise en circulation par le président George Bush de « guerre contre le terrorisme » va contribuer à justifier une intervention armée en Afghanistan comme réponse aux attentats du 11 septembre 2001 (p. 61, p. 156).

Le chapitre 3 met l'accent sur la dimension axiologique de l'objet des discours en tenant compte des routines rédactionnelles et du dispositif sémiotique du journal dans la configuration des différentes facettes de l'événement. L'auteure étudie le contexte dans la construction du sens, mettant l'accent sur l'importance des associations (analyse des cooccurrences) dans la configuration du sens d'un énoncé (chapitre 4). La nomination est également appréhendée dans sa dynamique interdiscursive comme l'attestent les marques d'interdépendances et de circulation entre différents discours et différents locuteurs. Le dialogisme est à la fois un principe de fonctionnement du langage qui se caractérise par l'imbrication, la reprise, la présence de différents discours, il a également une fonction sociale en tant que ressource langagière dans la construction discursive de l'événement (p. 126). Les récits des faits apparaissent ainsi façonnés par les discours qui les ont traversés, remettant en question la distinction entre faits et dires (chapitre 5). Le chapitre 6 s'intéresse aux références mémorielles associées à l'acte de nomination. Si des références à l'Ancien Régime et à la Révolution française ou à la lutte des classes servent de cadrage au récit du conflit des intermittents dans les deux quotidiens étudiés (p. 129), de nombreuses guerres sont convoquées dans le cas de la guerre en Afghanistan, comme la Première et la Seconde Guerre mondiale, la guerre du Kosovo ou la guerre du Golfe (p. 138). Le dernier chapitre qui synthétise les principales observations issues de l'analyse des corpus prolonge les interprétations en s'appuyant sur des travaux en sociologie des mouvements sociaux et en science politique afin de montrer la pertinence d'une approche en terme de profil lexico-discursif. La construction du sens social des deux événements étudiés souligne l'importance de l'hétérogénéité énonciative. Les discours autres interviennent à plusieurs niveaux, au niveau des reprises dans le texte et au niveau intertextuel par la mise en scène de dénominations et de descriptions concurrentes de l'événement, issues de différents actes énonciatifs. L'acte de nomination 
est central à deux titres dans la construction du sens de l'événement : en tant qu'opérateur de catégorisation et en tant qu'opérateur dialogique (p. 159).

Cet ouvrage dense s'avère néanmoins très didactique et il contribue, au même titre que les nombreux exemples à rendre accessibles aux non-linguistes le cheminement de la recherche. De nombreux aspects contribuent directement à une meilleure connaissance du travail de presse et à la sociologie du journalisme dans la construction du sens. Si ce travail intéressera à plus d'un titre les chercheurs en science politique et en sciences de l'information et de la communication, il intéressera tous les chercheur(e)s qui s'intéressent aux discours comme pratiques sociales ${ }^{2}$.

2. Cet ouvrage fait écho à deux autres publications collectives portant sur l'événement: Dire l'événement.

Langage, mémoire, société, dirigé par Danielle Londel, Sophie Moirand, Sandrine Reboul-

Touré et Licia

Reggiano (Presses

Sorbonne Nouvelle, 2013, 356 p.) et Les facettes de l'événement: des formes aux signes, dirigé par Elio Ballardini, Roberta Pederzoli, Sandrine Reboul-Touré et Geneviève TréguerFelten (Mediazoni, $\left.n^{\circ} 15,2013\right)$. 